\title{
Circulating Renin Angiotensin System in Childhood Chronic Renal Failure: Marked Increase of Angiotensin-(1-7) in End-Stage Renal Disease
}

\author{
ANA C. SIMÕES E SILVA, JOSÉ SILVÉRIO S. DINIZ, REGINA M. PEREIRA, SÉRGIO V. BRANT PINHEIRO, AND \\ ROBSON AUGUSTO S. SANTOS
}

Department of Pediatrics [A.C.S.S., J.S.S.D., S.V.B.P.], Faculty of Medicine, Universidade Federal de Minas Gerais, Belo Horizonte, MG, 30130-100, Brazil; Department of Physiology and Biophysics [R.M.P., R.A.S.S.], Institute of Biological Sciences, Universidade Federal de Minas Gerais, Belo Horizonte, MG, 31270-901, Brazil

\begin{abstract}
The aim of the present study was to evaluate plasma renin activity (PRA) and Angiotensin (Ang) levels [Ang I, Ang II and Ang-(1-7)] to examine the circulating Renin-Angiotensin System (RAS) in renal disease among children with different forms and stages of chronic renal failure (CRF). Subjects were divided as follows: 32 normotensive healthy subjects, 23 normotensive CRF subjects, 34 hypertensive CRF subjects and 21 subjects with endstage renal disease (ESRD). Radioimmunoassays for PRA (ngAngI/ $\mathrm{mL} / \mathrm{h})$ and angiotensin $(\mathrm{pg} / \mathrm{mL})$ measurements were performed on all subjects. PRA, Ang I, Ang II and Ang-(1-7) levels were significantly higher in hypertensive CRF subjects when compared with normotensive CRF and healthy subjects ( $p<0.05$ for all comparisons). No differences were observed between normotensive CRF and healthy subjects. ESRD subjects exhibited a dramatic increase in Ang-(1-7) (25-fold higher than control values). In hypertensive CRF subjects, treatment with angiotensin-converting enzyme inhibitors (ACEi) increased (1.4-fold) plasma Ang-(1-7) and decreased (2.4-fold) Ang II. In ESRD, the use of ACEi produced a similar (1.5-fold) elevation of Ang-(1-7), but no changes in plasma Ang II. Our data showed different circulating RAS profiles between hypertensive and in normotensive CRF subjects. Marked changes in plasma Ang-(1-7) were associated with the presence of hypertension and progression of kidney dysfunction. (Pediatr Res 60: 734-739, 2006)
\end{abstract}

$\mathrm{C}^{\mathrm{h}}$ hronic renal failure (CRF) in childhood frequently results from an identifiable renal disorder that evolves to endstage renal disease (ESRD) (1). Experimental and clinical data have shown that Renin-Angiotensin System (RAS) is clearly involved in the pathogenesis of renal diseases $(2,3)$. Acting on angiotensinergic type $1\left(\mathrm{AT}_{1}\right)$ receptors, Angiotensin (Ang) II induces progressive kidney injury via multiple mechanisms, including intraglomerular hypertension, sodium and water retention, stimulation of fibrogenic mediators, enhanced free radical formation, and contraction of mesangial cells $(3,4)$. Moreover, clinical trials have demonstrated that RAS inhibi-

Received February 1, 2006; accepted July 27, 2006.

Correspondence: Ana Cristina Simões e Silva, M.D., Ph.D., Rua Timbiras 1364, ap 1704 bloco A. Bairro Funcionários, Belo Horizonte, MG, Brazil, 30140-061; e-mail: acssilva@hotmail.com

Supported by FAPEMIG (Fundação de Amparo à Pesquisa do Estado de Minas Gerais, Brazil), CNPq (Conselho Nacional de Desenvolvimento Científico e Tecnológico, Brazil) and PRONEX (Programa de Grupos de Excelência-FINEP, Brazil). tors, angiotensin-converting enzyme inhibitors (ACEi) and $\mathrm{AT}_{1}$ receptor blockers reduce the rate of progression of CRF to $\operatorname{ESRD}(5,6)$.

The combination of physiologic and pharmacological approaches with molecular biology techniques has led to the identification of novel RAS components: Ang III, Ang IV and Ang-(1-7) $(7,8,9)$; the Ang IV binding site insulin-regulated aminopeptidase (10); angiotensin-converting enzyme 2 (ACE2), an homologue of the "classic" angiotensin-converting enzyme (ACE) $(11,12,13)$; and the Ang-(1-7) G protein-coupled receptor Mas (14). Among the novel RAS metabolites, Ang-(1-7) is particularly interesting. This heptapeptide is functionally linked to mechanisms that intrinsically regulate the function of the RAS $(7,8,9,15)$. Ang-(1-7) is formed from Ang II by prolylendopeptidase, prolyl-carboxipeptidase or ACE2 $(11,12,13)$ or directly from Ang I through hydrolysis by prolylendopeptidase and endopeptidase24.11 and metabolized by ACE to Ang-(1-5) (7,9). ACEi elevate Ang-(1-7) concentrations (16) by both increasing Ang I, the substrate for Ang-(1-7), as well as by preventing Ang-(1-7) degradation. Ang-(1-7) opposes the vascular and proliferative effects of Ang II and exerts complex renal actions $(7,8,9,15)$. Recent studies suggest that, at least in part, the beneficial effects of ACEi in heart and kidney diseases may be attributed to Ang-(1-7) (17).

Despite considerable experimental evidence regarding the role of Ang-(1-7) in cardiovascular and renal function (15), few data are available on humans. We have recently shown a selective increase of Ang-(1-7) in childhood essential hypertension, suggesting an imbalance of the vascular and/or renal turnover of this peptide (18). We have extended our previous study (18) by measuring plasma renin activity (PRA), Ang I, Ang II and Ang-(1-7) levels in pediatric subjects with different forms and stages of CRF to evaluate possible changes in the RAS profile. We divided the subjects into two groups

Abbreviations: ACE, angiotensin-converting enzyme; ACEi, angiotensinconverting enzyme inhibitors; ACE2, angiotensin-converting enzyme homologue; Ang, Angiotensin; AT $\mathbf{1}_{\mathbf{1}}$, angiotensin type 1; CCB, calcium channels blockers; CRF, chronic renal failure; ESRD, end-stage renal disease; PRA, plasma renin activity; RAS, Renin Angiotensin System

DOI: 10.1203/01.pdr.0000246100.14061.bc 
according to chronic use or nonuse of ACEi to verify the effect of treatment on the circulating RAS profile.

\section{SUBJECTS AND METHODS}

The present cross-sectional study used a convenience sample recruited from either the Pediatric Nephrology Unit or the Pediatric Primary Care Center of our institution.

Inclusion criteria. Subjects with CRF included only nondialysis pediatric subjects with an estimated GFR of $<60 \mathrm{~mL} / \mathrm{min} / 1.73 \mathrm{~m}^{2}$ as calculated by the Schwartz formula (19). The etiology of the kidney disease was established in all subjects. Subjects were divided regarding the presence or absence of arterial hypertension: hypertensive CRF, if both systolic and diastolic blood pressure were above the $95^{\text {th }}$ percentile for age, sex and height on at least three different occasions (20); and normotensive CRF, if systolic and diastolic blood pressure remained below the $90^{\text {th }}$ percentile for age, sex and height (20). The CRF group was treated only with conservative measures for the management of renal insufficiency, in which continuous clinical and laboratorial evaluation, nutritional support, water and electrolytes adjustments, vitamin, iron and alkali supplementation and blood pressure control are generally included when appropriate $(20,21)$.

ESRD was defined as a pronounced reduction of GFR $(<10 \mathrm{~mL} / \mathrm{min} / 1.73$ $\mathrm{m}^{2}$ ) for which renal replacement therapy was absolutely necessary. ESRD subjects were followed-up at the hemodialysis service of our institution.

The control group consisted of normotensive healthy age-matched subjects from our Pediatric Primary Care Center. Health status was determined through the subjects' medical history and either a parental report or self-report to rule out the presence of chronic or acute diseases. All subjects were submitted to a complete physical examination, blood measurements of urea and creatinine and an estimation of the GFR (GFR) using the Schwartz formula (19). Blood pressure was measured and classified according to Task Force recommendations (20). Only subjects with both systolic and diastolic pressure below the $90^{\text {th }}$ percentile according to age, sex and height were included. Urea and creatinine levels of the selected subjects were also within the normal range.

Exclusion criteria. Subjects without an identifiable renal disease were not included in the present study. Noncompliance to conservative treatment or hemodialysis program recommendations also excluded the subjects from the research protocol. Blood collection was suspended if the subject presented acute metabolic and/or hemodynamic disarrangements, such as hyperkalemia, acidosis, pulmonary edema or excessive interdialytic weight gain. Comorbidities such as diabetes, cardiac, pulmonary and neurologic diseases automatically excluded subjects.

Ethical aspects. The Ethics Committee of the Federal University of Minas Gerais approved the study. Informed consent was obtained from all included subjects and their parents. The research protocol did not interfere with any medical recommendations or prescriptions. Subject follow-up was guaranteed even in cases of refusal to participate in the study.

Study protocol. Blood sampling: Blood samples for PRA and plasma Ang measurements were obtained from healthy subjects, CRF (hypertensive and normotensive) and ESRD subjects on a single occasion, taking into account the inclusion and exclusion criteria for each group. Due to ethical reasons, no changes to the conservative CRF approach or dialysis prescriptions were made for study purposes. Blood samples were collected through peripheral venipuncture during the morning after a fasting period of $6 \mathrm{~h}$. All subjects rested in supine position for at least $15 \mathrm{~min}$ before blood pressure measurements and blood sampling. In hypertensive CRF subjects, these procedures were performed before the daily dosage of antihypertensive medications and, in ESRD subjects, procedures were performed just before the hemodyalisis session. Samples were withdrawn into two different sets of ice-cooled tubes, one containing $7.5 \%$ EDTA $(100 \mu \mathrm{L} / \mathrm{mL}$ of blood) for PRA determinations and the other containing a cocktail of protease inhibitors (18) for Ang measurements $(50 \mu \mathrm{L} / \mathrm{mL}$ of blood). Blood samples were centrifuged at $2000 \mathrm{~g}$ for $20 \mathrm{~min}$ at $4^{\circ} \mathrm{C}$, and plasma was stored at $-20^{\circ} \mathrm{C}(18)$.

Plasma extraction: Plasma samples were extracted using phenyl Bond-Elut cartridges (Analytichem International, Harbor City, CA), as previously described (18). The recovery of ${ }^{125}$ I-labeled Ang I, Ang II, and Ang-(1-7) was $79.2 \pm 2.3,86.9 \pm 0.8$ and $83.5 \pm 0.9 \%$, respectively.

Radioimmunoassays: PRA and the concentration of Ang I, Ang II and Ang-(1-7) were determined through radioimmunoassays, as previously described (18). Results were expressed as nanograms of Ang I generated per mL of plasma per hour (ngAngI/mL/h) for PRA and $\mathrm{pg} / \mathrm{mL}$ of plasma for Ang measurements.

Statistical analysis. Results were reported as mean \pm SD. Analysis of variance, followed by the Newman-Keuls test, was used for the comparison of mean values between the four selected groups. Comparisons between percentages of categorical variables were made by $\chi^{2}$ test. Hypertensive subjects were also divided according to chronic use or nonuse of ACEi. The unpaired Student $t$-test was used to compare PRA and Ang measurements in these groups. The level of significance was set at $p<0.05$.

\section{RESULTS}

Subject characteristics and casual measurements. Normotensive healthy subjects $(n=32)$ included 18 boys and 14 girls from 3.1-16.7 y. All children in this group had both systolic and diastolic pressure below the $90^{\text {th }}$ percentile (20). No medical or family history of renal disease or hypertension was detected. The mean values of urea and creatinine and the estimation of the GFR (19) were within the normal range (Table 1).

CRF subjects $(n=57)$ were divided into 23 subjects without hypertension (normotensive CRF) and 34 with hypertension (hypertensive CRF). Normotensive CRF subjects included 13 boys and 10 girls from $2.2-17.9 \mathrm{y}$, with both systolic and diastolic blood pressure within the $20^{\text {th }}$ and $90^{\text {th }}$ percentiles. They also presented a significant elevation of urea and creatinine levels, with a corresponding reduction of the GFR (Table 1). The causes of CRF in these subjects were uropathies $(65.2 \%)$, glomerulopathies (17.4\%), cystic renal diseases (13.1\%), and tubulopathies (4.3\%). Renal dysfunction in the subjects was treated

Table 1. Patient characteristics and blood measurements from control group, normotensive chronic renal failure (CRF) children, hypertensive CRF children, and end-stage renal disease patients

\begin{tabular}{|c|c|c|c|c|}
\hline $\begin{array}{c}\text { Characteristics and } \\
\text { measurements }\end{array}$ & $\begin{array}{l}\text { Control subjects } \\
\quad(n=32)\end{array}$ & $\begin{array}{l}\text { Normotensive CRF } \\
\text { children }(n=28)\end{array}$ & $\begin{array}{l}\text { Hypertensive CRF } \\
\text { children }(n=34)\end{array}$ & $\begin{array}{l}\text { ESRD patients } \\
\quad(n=21)\end{array}$ \\
\hline Sex (\%; male: female) & $56.2 / 43.8$ & $56.5 / 43.5$ & $47.1 / 52.9$ & $52.4 / 47.6$ \\
\hline Age (years) & $11.2 \pm 4.0$ & $12.0 \pm 4.0$ & $10.4 \pm 4.8$ & $13.0 \pm 3.3$ \\
\hline Diastolic BP (mmHg) & $62 \pm 17$ & $65 \pm 18$ & $112 \pm 28 *$ & $102 \pm 29 *$ \\
\hline Urea levels (mg/dL) & $22 \pm 4$ & $100 \pm 34 *$ & $99 \pm 42 *$ & $193 \pm 42 * \dagger$ \\
\hline Creatinine levels (mg/dL) & $0.5 \pm 0.1$ & $2.0 \pm 0.9^{*}$ & $2.3 \pm 1.3^{*}$ & $9.6 \pm 2.6 * \dagger$ \\
\hline Ang I (pg/ ml) & $26.4 \pm 13.7$ & $26.7 \pm 6.7$ & $171.8 \pm 85.4^{*}$ & $332.1 \pm 54.5^{* \dagger}$ \\
\hline Ang II (pg/ml) & $21.4 \pm 8.7$ & $22.4 \pm 9.1$ & $84.2 \pm 52.9 *$ & $113.2 \pm 25.8^{*}$ \\
\hline Ang-(1-7) $(\mathrm{pg} / \mathrm{ml})$ & $16.2 \pm 7.9$ & $18.6 \pm 6.8$ & $140.8 \pm 58.0^{*}$ & $419.2 \pm 82.2^{* \dagger}$ \\
\hline
\end{tabular}

$* p<0.05$ compared to control subjects; and $\dagger p<0.05$ for comparison between ESRD patients with other groups, using ANOVA followed by Newman-Keuls test.

$\S$ Glomerular filtration rate was estimated using the Schwartz formula. 
with conservative measures (21). Subjects did not receive any antihypertensive treatment before or at the time of blood sampling.

Hypertensive CRF subjects $(n=34)$ included 16 boys and 18 girls from 2.2-17.2 y. At diagnosis, all children had a significant elevation of urea and creatinine plasma levels and an equivalent decrease in GFR (Table 1). Both systolic and diastolic pressures were consistently above the $95^{\text {th }}$ percentile. The etiologies for primary renal disease in this group included glomerulopathies (44.2\%), cystic renal diseases (20.6\%), uropathies (17.6\%), tubulopathies (8.8\%), and hemolyticuremic syndrome $(8.8 \%)$. Antihypertensive therapy predominantly involved calcium channel blockers (CCB), either alone $(23.5 \%)$ or associated with ACEi $(29.4 \%)$ and with loop diuretics (17.7\%). Ten subjects needed 3 or more drugs for blood pressure control, including CCB plus ACEi plus loop diuretics with (14.7\%) or without minoxidil (14.7\%).

Subjects with ESRD $(n=21)$ included 11 boys and 10 girls from 5.9-16.8 y. All children had a marked elevation of urea and creatinine levels and a critical decrease of the estimated GFR (Table 1). All were submitted to hemodialysis and the majority was hypertensive $(85.7 \%)$. However, three subjects had both systolic and diastolic pressure within the normal range. Antihypertensive drugs were prescribed to the remaining 18 subjects, consisting of CCB plus ACEi (12 subjects), CCB plus loop diuretics (2 subjects) and $\mathrm{CCB}$ alone (4 subjects). $\mathrm{AT}_{1}$ antagonists were not used in hypertensive CRF and ESRD subjects.

Despite the same level of renal dysfunction, the causes of CRF differed significantly between normotensive and hypertensive subjects. Glomerular diseases were the most prevalent cause of hypertensive CRF (44.2\% vs. $17.4 \%$ in normotensive CRF subjects; $\chi^{2}=4.41, p<0.05$ ). In contrast, uropathies accounted for a significantly greater proportion of normotensive CRF subjects than hypertensive CRF subjects $(65.2 \%$ vs. $17.6 \% ; \chi^{2}=13.34, p<0.05$ ).

RAS profile in healthy subjects, CRF, and ESRD subjects. As shown in Table 1, no statistical differences were detected through the comparison of RAS measurements obtained respectively from normotensive CRF subjects and healthy subjects. Hypertensive CRF subjects exhibited a significant elevation of PRA, Ang I, Ang II and Ang-(1-7) when compared with normotensive CRF subjects or healthy subjects ( $p<0.05$ for all comparisons, Fig. 1).

Plasma Ang-(1-7) levels were markedly elevated in ESRD subjects $(p<0.01$ when compared with healthy subjects, normotensive and hypertensive CRF, Fig. 1). In contrast, Ang II levels did not differ from hypertensive CRF subjects, although they were significantly elevated in comparison to control group and normotensive CRF (Table 1). In ESRD subjects, as with Ang-(1-7), Ang I levels were significantly higher than in all other groups (Fig. 1). Table 1 shows that the mean levels of PRA in ESRD subjects were significantly higher than control values and normotensive CRF subjects, but lower than hypertensive CRF children $(p<0.05$ for all comparisons).

Ratios between Ang-(1-7) and Ang I levels and between Ang II and Ang I in all groups studied are displayed in Fig. 2. The Ang-(1-7)/Ang I ratio was significantly increased in ESRD subjects $(1.27 \pm 0.61)$ in comparison to other groups in

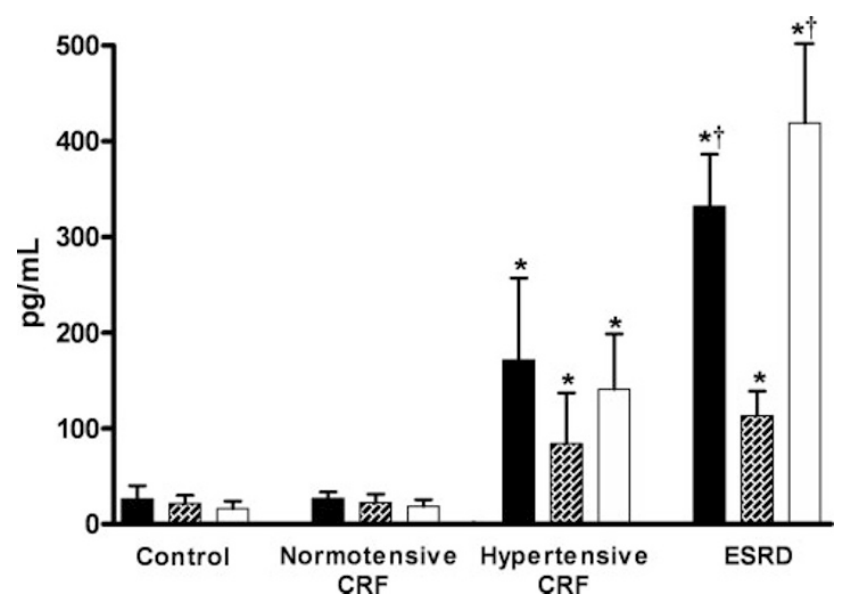

Figure 1. Ang I (black bars), Ang II (dashed bars) and Ang-(1-7) (white bars) plasma levels in normotensive healthy children (control) $(n=32)$, normotensive chronic renal failure (CRF) $(n=23)$, hypertensive CRF $(n=$ $34)$, and end-stage renal disease (ESRD) $(n=21)$ subjects. Data are expressed as means $\pm \mathrm{SD} * p<0.05$ compared with normotensive control subjects and ${ }^{\dagger} p<0.05$ for comparison between ESRD subjects with other groups, using ANOVA followed by the Newman-Keuls test.

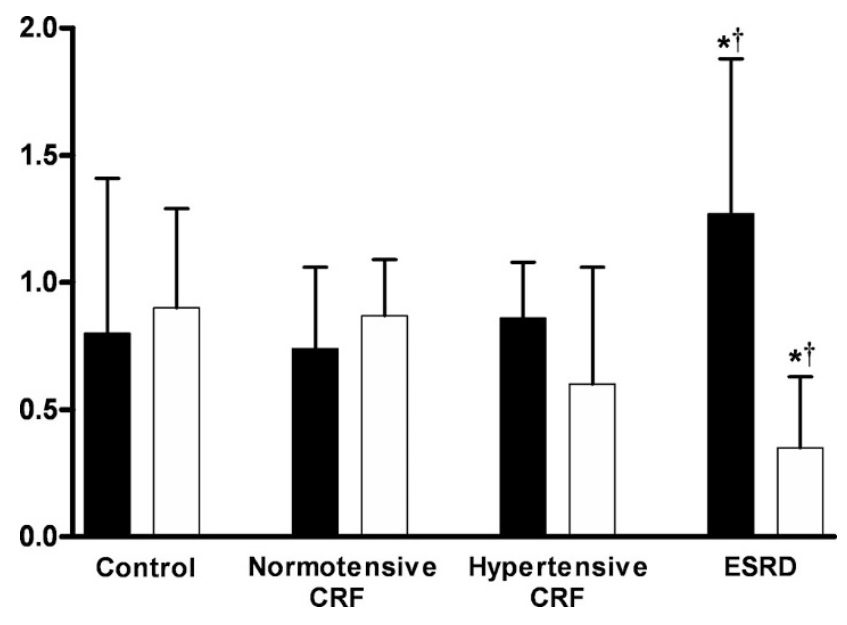

Figure 2. Ratios between Ang-(1-7) and Ang I plasma levels (black bars) and between Ang II and Ang I plasma levels (white bars) in normotensive healthy children (control) $(n=32)$, normotensive chronic renal failure (CRF) $(n=23)$, hypertensive CRF $(n=34)$, and end-stage renal disease (ESRD) $(n=21)$ subjects. Data are expressed as means $\pm \mathrm{SD} * p<0.05$ compared with normotensive control subjects and ${ }^{\dagger} p<0.05$ for comparison between ESRD subjects with other groups, using ANOVA followed by the NewmanKeuls test.

which the results were similar (control: $0.80 \pm 0.61$; normotensive CRF: $0.74 \pm 0.32$; and hypertensive CRF: $0.86 \pm$ 0.22 , Fig. 2). However, the Ang II/Ang I ratio was significantly reduced in ESRD $(0.35 \pm 0.28)$ when compared with all other groups (control: $0.90 \pm 0.39$; normotensive CRF: $0.87 \pm 0.39$; and hypertensive CRF: $0.60 \pm 0.46$, Fig. 2). Although higher than in ESRD subjects, the Ang II/Ang I ratio in hypertensive CRF subjects was significantly lower than in normotensive CRF and healthy subjects.

As mentioned above, only 3 of the 21 ESRD subjects did not require antihypertensive medications. PRA [0.8 $\pm 0.1 \mathrm{vs}$. $1.3 \pm 0.2]$, Ang I [230.4 \pm 47.6 vs. $349.0 \pm 33.1]$ and Ang-(1-7) [271.4 \pm 32.9 vs $443.8 \pm 58.5]$ were lower than values obtained in hypertensive ESRD subjects $(p<0.05$ for 
all comparisons). Ang II levels were slightly but not significantly reduced in these subjects when compared with hypertensive ESRD subjects $(89.3 \pm 9.7 v s .117 .2 \pm 25.6 \mathrm{pg} / \mathrm{mL})$. However, these three normotensive ESRD subjects presented a significant elevation of PRA and angiotensin levels when compared with healthy subjects and normotensive CRF subjects $(p<0.05)$.

$R A S$ profile in the presence or absence of $A C E$ inhibition. Data from hypertensive CRF and ESRD subjects were divided according to chronic use or nonuse of ACE inhibition to evaluate the influence of pharmacological RAS blockade on circulating Ang levels.

As shown in Table 2, chronic treatment with ACEi in hypertensive CRF subjects increased PRA, Ang I and Ang(1-7) levels and decreased circulating Ang II as compared with subjects who did not receive ACEi $(p<0.05$ for all comparisons). However, although reduced, circulating levels of Ang II remained approximately 2.4-fold higher than mean values of healthy subjects and normotensive CRF subjects ( $p<0.05$ for both comparisons).

Results obtained with ESRD subjects were even more impressive, as the blockade of ACE was unable to change plasma levels of Ang II (Table 2), which continued to be 5-fold higher than control values. In contrast, chronic use of ACEi in ESRD subjects produced significant elevations of PRA, Ang I and Ang-(1-7) levels when compared with subjects not treated with ACEi ( $p<0.05$ for all comparisons). The percentage of increase in Ang-(1-7) levels (50\%) was similar in ESRD and hypertensive CRF subjects.

It is noteworthy that hypertensive CRF subjects not receiving any kind of RAS blockade (Table 2) exhibited significantly higher levels of all RAS components than normotensive CRF and control subjects (Table 1). The same result was obtained with ESRD subjects not treated with ACEi. Moreover, Ang-(1-7) levels (325.3 \pm 67.1$)$ remained significantly elevated even when compared with the entire group of hypertensive CRF subjects.

\section{DISCUSSION}

The study clearly demonstrates different RAS profiles in childhood CRF, with marked increases of Ang-(1-7) in the presence of hypertension and ESRD. Hypertensive CRF subjects presented an overall RAS activation that significantly differed from normotensive CRF subjects with the same level of GFR. These data agree with previous studies that showed a significant elevation of PRA and Ang II levels in adults with hypertensive renal diseases $(22,23)$.

However, plasma Ang-(1-7) levels had not been evaluated in childhood CRF until the present study. We detected a significant increase in this heptapeptide among hypertensive CRF children and an even more pronounced elevation among ESRD subjects. On the other hand, Ang-(1-7) levels were similar in normotensive CRF subjects and in healthy subjects, despite the significant difference in GFR. We have recently reported significant differences in Ang II and Ang-(1-7) plasma levels among pediatric subjects with renovascular disease and essential hypertension (18). In renovascular disease, plasma levels of Ang II were higher than Ang-(1-7) and the surgical correction of renal artery stenosis produced a return of circulating angiotensins to healthy subject values. In contrast, essential hypertensive subjects exhibited a selective and blood-independent elevation of plasma Ang-(1-7) (18). Taken together, our results show changes in the RAS profile according to differences in hypertensive states and the degree of renal dysfunction. These findings do not allow any mechanistic inferences on the precise meaning of Ang-(1-7) elevation, although some possibilities could be raised.

Firstly, we obviously cannot rule out the possibility that plasma Ang-(1-7) elevation was only caused by a significant reduction of renal excretion of the peptide in ESRD subjects. However, a comparison between circulating Ang II in ESRD subjects and hypertensive CRF subjects shows no significant differences, clearly contrasting with the results obtained for Ang-(1-7). Thus, another attractive explanation is a disturbance in the metabolism of Ang-(1-7) among pediatric subjects with ESRD, resembling our previous data in childhood essential hypertension (18). The increased ratio between Ang(1-7) and Ang I plasma levels concomitant with the lower Ang II/Ang I ratio in these subjects when compared with other groups substantiates this possibility, suggesting that Ang(1-7) could be the major RAS product in ESRD children. The metabolic pathways in these subjects remain unknown.

It should also be pointed out that Ang-(1-7) is present in the kidney at concentrations that are comparable to Ang II and also exerts complex renal effects $(7,9,15,17,24-26)$. The diuretic/natriuretic action of Ang-(1-7) has been described in several in vitro and in vivo experimental models $(9,25)$. On the other hand, our group and others have shown Ang-(1-7) to produce an antidiuretic/antinatriuretic effect (27-32). Differences between species, local and systemic concentrations of

Table 2. Renin Angiotensin System (RAS) circulating profile [PRA-plasma renin activity, Ang I, Ang II and Ang-(1-7) levels] in hypertensive chronic renal failure $(C R F)$ and end-stage renal disease (ESRD) patients, according to chronic use or non-use of angiotensin-converting enzyme inhibitors (ACEi) Hypertensive CRF children

\begin{tabular}{l} 
RAS measurements \\
\hline PRA $(\mathrm{ng}$ AngI/mL/h) \\
Ang I $(\mathrm{pg} / \mathrm{mL})$ \\
Ang II $(\mathrm{pg} / \mathrm{mL})$ \\
Ang- $(1-7)(\mathrm{pg} / \mathrm{mL})$
\end{tabular}

ESRD patients

\begin{tabular}{cc}
\hline $\begin{array}{c}\text { With ACEi } \\
(n=12)\end{array}$ & $\begin{array}{c}\text { Without ACEi } \\
(n=9)\end{array}$ \\
$1.31 \pm 0.25^{*}$ & $0.94 \pm 0.27$ \\
$353.53 \pm 67.06^{*}$ & $278.37 \pm 63.35$ \\
$113.01 \pm 25.34$ & $113.80 \pm 29.40$ \\
$456.75 \pm 52.39 *$ & $325.31 \pm 67.06$
\end{tabular}

$* p<0.05$ for the comparison of each RAS measurement in the presence or absence of ACEi therapy using unpaired Student $t$-test. 
Ang-(1-7), nephron segment, level of RAS activation and sodium and water status may be responsible for the discrepancy in results concerning the renal effects of Ang-(1-7) $(9,15,24-26)$. We must consider the probability that changes in plasma volume and osmolality interfered with the state of RAS activation and thus contributed toward different levels of circulating angiotensins in our subjects, especially in the ESRD group.

We also obtained a preliminary evaluation of RAS metabolism through a comparison of Ang peptides in the presence or absence of ACE inhibition. Treatment with ACEi produced diverse patterns of plasma angiotensins in hypertensive CRF and ESRD subjects. Our data are in agreement with the plasma and urinary elevation of Ang-(1-7) observed in hypertensive rats treated with lisinopril (33) and in essential hypertensive adults receiving captopril (34). In hypertensive CRF subjects, ACE inhibition partially reduced Ang II levels, though they remained two times higher than the control values. In ESRD subjects, however, treatment with ACEi did not change Ang II levels, which continued to be five times greater than the control values. In contrast, circulating Ang-(1-7) was similarly increased with ACE inhibition in both groups. The clinical relevance of these findings is as yet unclear. However, the fact that ACE inhibition was unable to produce a complete blockade of Ang II formation speaks to the possibility that the harmful effects of Ang II on the kidney, heart and blood vessels $(3,4)$ remain uncontrolled under ACE inhibition. Accordingly, the double blockade of RAS with the concomitant administration of $\mathrm{ACEi}$ and $\mathrm{AT}_{1}$ receptor blockers can minimize or even overcome the escape observed with the singlesite blockade (35). Indeed, Ang-(1-7) is significantly increased with both types of RAS blockade $(8,9,16,17)$, suggesting a role in the double blockade mechanisms $(17,35)$. The antihypertensive response to ACEi in animal models was reduced by $30-40 \%$ when Ang-(1-7) synthesis was prevented (33). In keeping with these observations, we can hypothesize that an alteration to the RAS metabolism or Ang-(1-7) activity may contribute toward the evolution of hypertension and human renal diseases.

We are aware of the limitations associated with the crosssectional design of our study. The main possible weakness was the use of a convenience sample, which makes homogeneity among the selected groups very difficult to obtain. Thus, there were significant differences in the primary diagnosis and medication exposure between the CRF and ESRD populations. Nevertheless, some aspects of the study may increase the strength of our findings, such as the utilization of strictly defined inclusion and exclusion criteria and the wellestablished protocol for the measurements of PRA and angiotensins (18).

In summary, we have shown different circulating RAS profiles in CRF subjects as preliminary evidence for the role of Ang-(1-7) in hypertension associated with kidney disease and in ESRD. Although, it is as yet unknown if the elevation of Ang-(1-7) occurs as a compensatory mechanism that opposes the harmful renal and cardiovascular effects of Ang II; or whether, due to a major unbalance in the RAS metabolism,
Ang-(1-7) at supra physiologic concentrations could act as a mediator of renal dysfunction. Further studies are obviously needed to clarify these possibilities.

\section{REFERENCES}

1. Ardissino G, Dacco V, Testa S, Bonaudo R, Claris-Appiani A, Taioli E, Marra G, Edefonti A, Sereni F 2003 Epidemiology of chronic renal failure in children: Data from the italkid project. Pediatrics 111:E382-E387

2. Shatat IF, Flynn JT 2005 Hypertension in children with chronic kidney disease. Adv Chronic Kidney Dis 12:378-384

3. Nangaku M, Ohse T, Tanaka T, Kojima I, Fujita T 2005 Renoprotection with anti-hypertensives: Reduction of proteinuria and improvement of oxygenation via inhibition of the renin-angiotensin system. Curr Hypertens Rev 1:67-76

4. Kim S, Iwao H 2000 Molecular and cellular mechanisms of Angiotensin II-mediated cardiovascular and renal diseases. Pharmacol Rev 52:11-34

5. Remuzzi G, Ruggeneti P, Perico N 2002 Chronic renal disease: Renoprotective benefits of RAS inhibition. Ann Intern Med 136:604-615

6. Zaman MA, Oparil S, Calhoun DA 2002 Drugs targeting the renin angiotensin aldosterone system. Nat Rev Drug Discov 1:621-636

7. Santos RA, Campagnole-Santos MJ, Andrade SP 2000 Angiotensin-(1-7): An update. Regul Pept 91:45-62

8. Carey RM, Siragy HM 2003 Newly recognized components of the renin-angiotensin system: Potential roles in cardiovascular and renal regulation. Endocr Rev 24:261271

9. Chappell MC, Modrall JG, Diz DI, Ferrario CM 2004 Novel aspects of the renal Renin-Angiotensin System: Angiotensin-(1-7), ACE2 and blood pressure regulation. Contrib Nephrol 143:77-89

10. Albiston AL, McDowall SG, Matsacos D, Sim P, Clune E, Mustafa T, Lee J, Mendelsohn FA, Simpson RJ, Connolly LM, Chai SY 2001 Evidence that the angiotensin IV $\left(\mathrm{AT}_{4}\right)$ receptor is the enzyme insulin-regulated aminopeptidase. J Biol Chem 276:48623-48626

11. Donoghue M, Hsieh F, Baronas E, Godbout K, Gosselin M, Stagliano N, Donovan M, Woolf B, Robison K, Jeyaseelan R, Breitbart RE, Acton S 2000 A novel angiotensin-converting enzyme-related carboxypeptidase (ACE2) converts angiotensin I to angiotensin 1-9. Circ Res 87:E1-E9

12. Tipnis SR, Hooper NM, Hyde R, Karran E, Christie G, Turner AJ 2000 A human homolog of angiotensin-converting enzyme. Cloning and functional expression as a captopril-insensitive carboxypeptidase. J Biol Chem 275:33238-33243

13. Eriksson U, Danilczyk U, Penninger JM 2002 Just the beginning: Novel functions for angiotensin-converting enzymes. Curr Biol 12:R745-R752

14. Santos RA, Simões e Silva AC, Maric C, Silva DR, Machado RP, Buhr I, HeringerWalther S, Pinheiro SV, Lopes MT, Bader M, Mendes EP, Lemos VS, CampagnoleSantos MJ, Schultheiss HP, Speth R, Walther T 2003 Angiotensin-(1-7) is an endogenous ligand for the G-protein coupled receptor Mas. Proc Natl Acad Sci USA 100:8258-8263

15. Simões e Silva AC, Pinheiro SV, Pereira RM, Ferreira AJ, Santos RA 2006 The therapeutic potential of angiotensin-(1-7) as a novel renin angiotensin system mediator. Mini Rev Med Chem 6:603-609

16. Campbell DJ 2003 The renin angiotensin and kallikrein kinin systems. Int J Biochem Cell Biol 35:784-791

17. Santos RA, Ferreira AJ, Pinheiro SV, Sampaio WO, Touyz R, Campagnole-Santos MJ 2005 Angiotensin-(1-7) and its receptor Mas as a potential target for new cardiovascular drugs. Expert Opin Investig Drugs 14:1019-1031

18. Simões e Silva AC, Diniz JS, Regueira-Filho A, Santos RA 2004 The renin angiotensin system in childhood hypertesion: Selective increase of angiotensin-(1-7) in essential hypertension. J Pediatr 145:93-98

19. Schwartz GJ, Haycock GB, Edelmann CM, Spitzer A 1976 A simple estimate of glomerular filtration rate in children derived from body lenght and plasma creatinine. Pediatrics 58:259-263

20. National High Blood Pressure Education Program Working Group on High Blood Pressure in Children and Adolescents 2004 The fourth report on the diagnosis, evaluation and treatment of the high blood pressure in children and adolescents. Pediatrics 114:555-576

21. Fine RN, White DA, Boydstun II 2004 Conservative management of chronic renal insufficiency. In: Avner ED, Harmon WE, Niaudet P (eds) Pediatric Nephrology $5^{\text {th }}$. Lippincott Williams \& Wilkins, Philadelphia, pp 1291-1311

22. Sinclair AM, Isles CG, Brown I, Cameron H, Murray BD, Robertson JW 1987 Secondary hypertension in a blood pressure clinic. Arch Intern Med 147:1289-1293

23. Martínez-Maldonado M 1998 Hypertension in end-stage renal disease. Kidney Int Suppl 54:S67-S72

24. Simões e Silva AC, Baracho NC, Passaglio KT, Santos RA 1997 Renal actions of angiotensin-(1-7). Braz J Med Biol Res 30:503-513

25. Chappell MC, Iyer SN, Diz DI, Ferrario CM 1998 Antihypertensive effects of angiotensin-(1-7). Braz J Med Biol Res 31:1205-1212

26. Santos Dos Santos RA, Passaglio KT, Pesquero JB, Bader M, Simões e Silva AC 2001 Interactions between Angiotensin-(1-7), Kinins and angiotensin ii in kidney and blood vessels. Hypertension 38:660-664 
27. Garcia NH, Garvin JL 1994 Angiotensin-(1-7) has a biphasic effect on fluid absorption in the proximal straight tubule. J Am Soc Nephrol 5:1133-1138

28. Vallon V, Richter K, Heyne N, Osswald H 1997 Effect of intratubular application of angiotensin-(1-7) on nephron function. Kidney Blood Press Res 20:233-239

29. Santos RA, Simões e Silva AC, Magaldi AJ, Khosla MC, Cesar KR, Passaglio KT, Baracho NC 1996 Evidence for a physiological role of Angiotensin-(1-7) in the control of hydroelectrolyte balance. Hypertension 27:875-884

30. Simões e Silva AC, Bello AP, Baracho NC, Khosla MC, Santos RA 1998 Diuresis and natriuresis produced by long term administration of a selective Angiotensin(1-7) antagonist in normotensive and hypertensive rats. Regul Pept 74:177-184

31. Magaldi AJ, Cesar KR, Araújo M, Simões e Silva AC, Santos RA 2003 Angiotensin(1-7) stimulates water transport in rat inner medullary collecting duct: Evidence for novel involvement of Vasopressin $V_{2}$ receptor. Pfulgers Arch 447:223-230
32. Pinheiro SV, Simões e Silva AC Sampaio WO, Paula RD, Mendes EP, Bontempo ED, Pesquero JB, Walther T, Alenina N, Bader M, Bleich M, Santos RA 2004 The nonpeptide AVE0991 is an angiotensin-(1-7) receptor Mas agonist in the mouse kidney. Hypertension 44:490-496

33. Iyer SN, Ferrario CM, Chappell MC 1998 Angiotensin-(1-7) contributes to the antihypertensive effects of blockade of the renin-angiotensin system. Hypertension 31:356-361

34. Luque M, Martin P, Martell N, Fernandez C, Brosnihan KB, Ferrario CM 1996 Effects of captopril related to increased levels of prostacyclin and angiotensin-(1-7) in essential hypertension. J Hypertens 14:799-805

35. Azizi M, Ménard J 2004 Combined blockade of the Renin Angiotensin System with Angiotensin-converting enzyme inhibitors and Angiotensin II type 1 receptor antagonists. Circulation 109:2492-2499 